\title{
Ameliorating effects of Garcinia mangostana Linn pericarp extract on hepatic antioxidants in Diethyl nitrosamine (DEN) induced Hepatocellular Carcinoma (HCC)
}

\author{
Vishnu Priya Veeraraghavan', Surapaneni Krishna Mohan ${ }^{2}$, Mallika Jainu ${ }^{3}$ and Chandra Sada Gopan \\ Venkipuram Seshadri ${ }^{4}$
}

${ }^{1}$ Department of Biochemistry, Saveetha Dental College \& Hospital, Saveetha University, Velappanchavadi, Chennai -600077 , Tamilnadu, INDIA.

2Department of Biochemistry, Saveetha Medical College \& Hospital, Faculty of Medicine, Saveetha University, Saveetha Nagar, Thandalam, Chennai - 602 105, Tamilnadu, INDIA.

${ }^{3}$ Department of Biomedical Engineering, SSN Engineering College, OMR, Klavakkam, Chennai - 603 110, Tamilnadu, INDIA. ${ }^{4}$ Department of Biochemistry, Priyadarshini Dental College \& Hospital, No.1, V.G.R Gardens, V.G.R Nagar, Pandur - 631 203,

Thiruvallur Taluk \& District, Tamilnadu, INDIA.

\begin{abstract}
Background: The extract of Garcinia mangostana Linn., (GM) has demonstrated antioxidant, anti-tumoral, anti-allergic, anti-inflammatory, antibacterial and antiviral activities and is widely used as a therapeutic drug in South-East Asia. Methods: The methanolic extract of GM fed orally to a set of Wistar rats in which hepatic carcinoma is induced with the help of $\mathrm{N}$-diethylnitrosamine (DEN) forms the heart of this study. Four groups of 6 rats each is used for the study. Group I: Normal rat received distilled water; Group II: Rats induced with DEN; Group III: Rats induced with DEN and cotreated with GM extract; Group IV: Rats treated with GM extract alone. Clinical chemistry, organ weights, nodular incidence, enzymatic and antioxidant analysis were carried out. Results: It is observed that the rats fed only with DEN developed tumor in the liver cells and those fed with the drug showed increased resistance to the spread and development of hepatic carcinoma. The non enzymatic antioxidant such as GSH and antioxidant enzymes such as catalase and SOD were decreased significantly with simultaneous LPO increase in group 2 rats, compared to that of control group. The carcinoma treated with GM extract showed significant increase in the levels of antioxidants viz. GSH, catalase, SOD and decrease in LPO when compared with that of group 2 rats. Conclusion: The results of this study concludes that the pericarp extract of GM exhibits profound anti-oxidant abilities which reduces the levels of lipid peroxides and increases the levels of glutathione, SOD and CAT to near normal performance of the liver. Further analysis of the antioxidant enzymatic study showed that the drug had positive effects in inhibiting hepatic cancer.
\end{abstract}

Key words: Antioxidants, Catalase, Diethylnitrosamine, Garcinia mangostana Linn, Hepatocellular carcinoma, Liver Marker enzymes, Lipid peroxidation, Marker enzymes, Superoxide dismutase.
Submission Date : 12-02-2015 Revision Date : :02-04-2015 AcceptedDate : :23-04-2015

DOI: 10.5530/ijper.49.4.11 Correspondence Address Dr. Surapaneni Krishna Mohan

Associate Professor \& Vice Principal, Department of Biochemistry, Saveetha Medical College and Hospital,

Faculty of Medicine, Saveetha University,

Saveetha Nagar, Thandalam, Chennai-602105, Tamilnadu, INDIA.

E-mail:krishnamohan.surapaneni@gmail.com

\section{INTRODUCTION}

Hepatic carcinoma is the fifth most widely spread type of tumor and death incidents due to this profoundly observed in developing countries where most of the world's population is concentrated. Hepatic carci- noma is attributed to a variety of causative agents like food, ${ }^{1}$ genetic and environmental factors $^{2}$ of which consumption of alcohol and high fat diet becomes the most pathogenic.

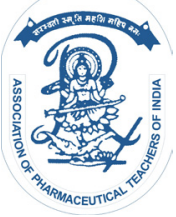

www.ijper.org 


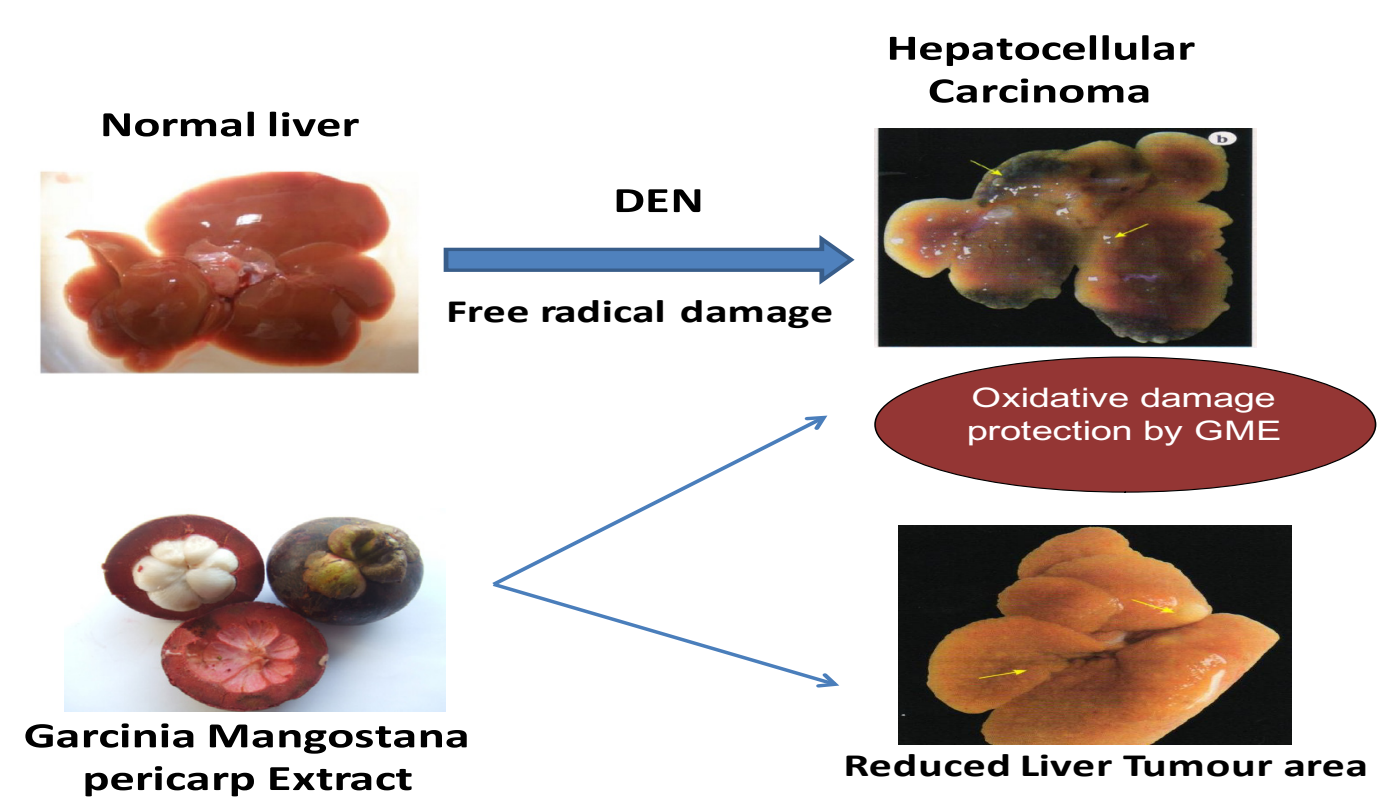

Graphical Abstract

Carcinogenesis occurs and develops in 3 steps. Initiation, where the normal cells in the organ turn carcinogenic. Promotion, in this stage the surrounding cells of the newly developed carcinogenic cell also become tumor cells. Progression, this is the most critical stage where the cancer cells break from the parent organ, mixes into the circulatory system, flows and settles somewhere else in the body and causes tumor in that particular organ. ${ }^{3}$

For experimental studies cancer is induced on rat models by a powerful carcinogenic chemical widely in use N-Diethyl nitrosamine (DEN). DEN is the important environmental carcinogens, ${ }^{4}$ abundantly present in processed food ${ }^{5}$ like meat and milk, and alcohol. ${ }^{6}$ It is also formed due to various in vivo conditions occurring due to physiological changes. ${ }^{7} \mathrm{DEN}$ induces pathogenic lesions in rat that is similar to many types of malignant and benign tumors observed in human beings. ${ }^{8}$ The extent to which the cells of the liver are affected by DEN is based on the dose of the chemical administered to the animal under examination. At very low doses of $10 \mathrm{mg} /$ $\mathrm{kg}$ body weight, DEN induces only initiation of carcinogenesis and hepatic fibrosis. ${ }^{9}$ As the dose levels increase above $30 \mathrm{mg} / \mathrm{kg}$ body weight cancer is well grown and promotion and progression of the tumor takes place. ${ }^{10}$

The growth and promotion of cancer has been attributed to the free radicals generated. This has been justified through various clinical trials. ${ }^{11}$ Free radicals promote oxidative stress to the cells that enhances tumor growth. ${ }^{12,13}$ Oxidants alter the functionality of the cell majorly the changes to genes that generate the mitogenic signals to the carcinogen exposed cells which bring about tumor promotion, also initiates toxicity into surrounding normal cells. ${ }^{14,15,16}$ Anti-oxidants delay or inhibit a wide variety of biochemical processes of tumor promotion. They protect the point of attack of the free radicals and prevent the mutation of DNA and damage of cell membrane thereby inhibiting the initiation of carcinogenesis. Anti-oxidants perform in a more sequential pattern of multi-layer prevention, interception and repairing the damaged cell membranes. ${ }^{17,18,19,20}$ Chemoprevention of cancer is very important and has significant effects from the plant extracts. ${ }^{21}$

Garcinia mangostana, colloquially known simply as "the mangosteen", is a tropical evergreen tree, and is widely utilized nowadays as a powerful drug in the South East Asian countries, as it has a combination of nutrient rich, therapeutic and appealing features like fragrance, visual attraction, taste and very high anti-oxidant strength. ${ }^{22} \mathrm{It}$ has high potential to reduce the risk of many human ailments ${ }^{23}$ as it contains polyphenols, that can be extracted to phytochemical values and is administered orally to overcome dysentery and applied on eczema and skin related problems as an ointment. These are very rich in xanthones, that possess excellent anti-oxidant characteristics and are the most valuable natural substances that has numerous bio-protective properties. They play a very important role in the protection of liver, intestine and bone and cartilage functions. ${ }^{24}$

\section{MATERIALS AND METHODS}

\section{Test drug and Chemicals}

G. mangostana pericarp extracts powder as obtained from Avasthagen Company, California, USA as a compliment and is applied for the present investigation. All other chemicals used were of analytical grade. 


\section{Table 1: Study of G. mangostana extract on body weight and liver weight}

\begin{tabular}{|c|c|c|c|}
\hline Group & $\begin{array}{c}\text { Initial weight } \\
\text { (in grams) }\end{array}$ & $\begin{array}{c}\text { Final body weight } \\
\text { (in grams) }\end{array}$ & $\begin{array}{c}\text { Liver weight } \\
\text { (in grams) }\end{array}$ \\
\hline Control & $180 \pm 1.1$ & $200 \pm 2.0$ & $4.72 \pm 0.39$ \\
\hline DEN alone & $180 \pm 1.5^{*}$ & $165 \pm 1.5^{*}$ & $8.75 \pm 0.53^{*}$ \\
\hline DEN + GME (400 $\mathbf{~ m g / k g ~ b . w t ) ~}$ & $180 \pm 1.6^{\mathrm{a}}$ & $190 \pm 1.2^{\mathrm{a}}$ & $5.12 \pm 0.30^{\mathrm{a}}$ \\
\hline GME alone (400 $\mathbf{~ m g / k g ~ b . w t )}$ & $180 \pm 1.4$ & $200 \pm 1.7$ & $4.71 \pm 0.42$ \\
\hline
\end{tabular}

Results are expressed as mean \pm S.E.M, $n=6$. ${ }^{*} \mathrm{P}<0.001$, statistically significant as compared with control rats and ${ }^{a} \mathrm{P}<0.001$, statistically significant as compared with DEN alone treated animals.

\begin{tabular}{|c|c|c|c|}
\hline \multicolumn{2}{|c|}{ Table 2: Effect of G. mangostana extract on tumor hepatic nodules } \\
\hline Group & $\begin{array}{c}\text { No. of hepatic } \\
\text { nodules }\end{array}$ & $\begin{array}{c}\text { No. of rats with } \\
\text { nodules }\end{array}$ & $\begin{array}{c}\text { Size of nodules } \\
>1-3 ~ \mathbf{~ m m}\end{array}$ \\
\hline Control & - & n.d & n.d \\
\hline DEN alone & $195^{*}$ & $6 / 6$ & $44.5 \pm 2.6^{*}$ \\
\hline DEN + GME (400 $\mathbf{~ g / k g ~ b . w t ) ~}$ & $65^{\mathrm{b}}$ & $4 / 6$ & $22.6 \pm 1.2^{\mathrm{b}}$ \\
\hline GME alone & - & n.d & n.d \\
\hline
\end{tabular}

Results are expressed as mean \pm S.E.M, $n=6$. * $P<0.001$, statistically significant as compared with control rats and ${ }^{\mathrm{b}} \mathrm{P}<0.001$ and $\mathrm{PP}<0.05$, statistically significant as compared with Group 2.

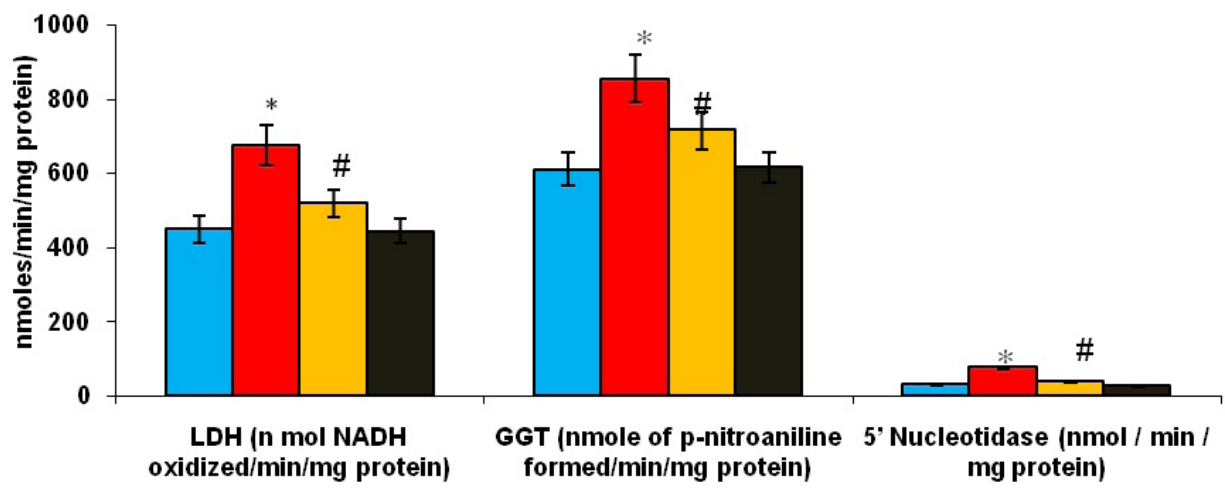

\begin{tabular}{|ll|}
\hline QControl & aDen Alone \\
QDEN+GML & QGML alone \\
\hline
\end{tabular}

A

Figure 1A: Levels of serum liver marker enzymes in control and experimental rats Results are expressed as mean \pm S.E.M, $n=6$. ${ }^{*} P<0.001$, statistically significant as compared with control rats and ${ }^{\#}<<0.001$ statistically significant as compared with $D E N$ control group.

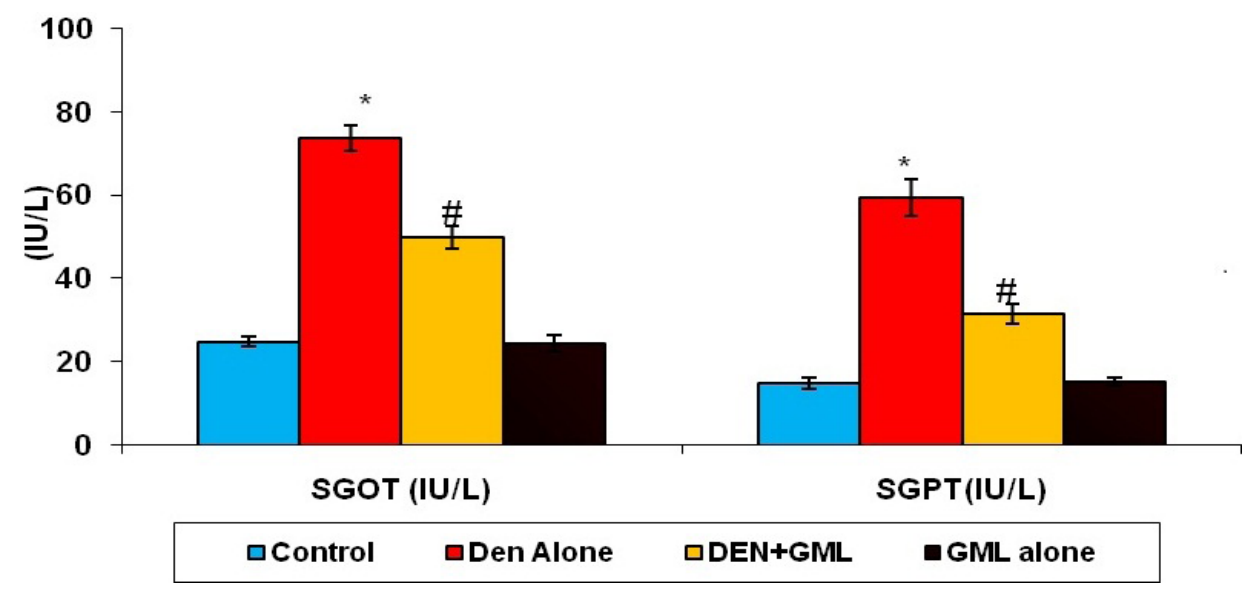

B

Figure 1B: Levels of serum liver marker enzymes in control and experimental rats

Results are expressed as mean $\pm S . E . M, n=6 .{ }^{*} P<0.001$, statistically significant as compared with control rats and ${ }^{*} P<0.001$ statistically significant as compared with $D E N$ control group. 


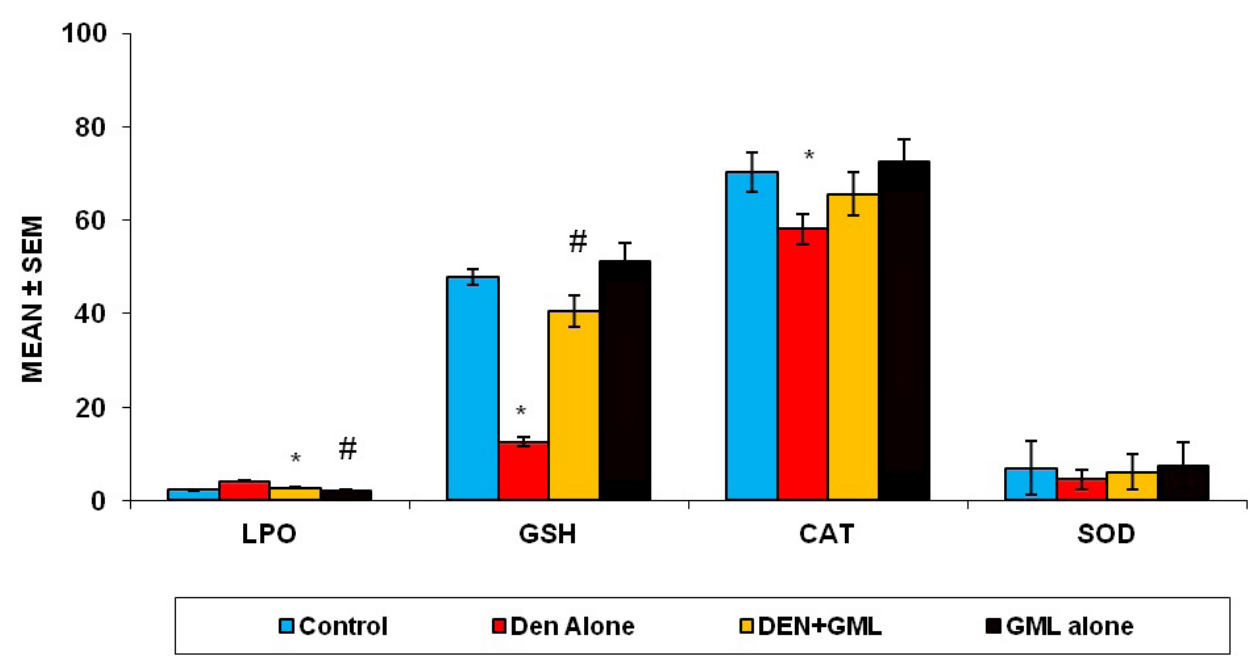

Figure 2: Activities of enzymic antioxidant and lipid peroxidation levels in liver tissue in control and experimental rats Results are expressed as mean \pm S.E.M, $n=6$. ${ }^{*} P<0.001$, statistically significant as compared with control rats and ${ }^{*} P<0.001$ statistically significant as compared with $D E N$ control group. SOD- unit/min/mg protein; CAT - $\mu$ moles of $\mathrm{H}_{2} \mathrm{O}_{2} / \mathrm{min} / \mathrm{mg}$ protein; $\mathrm{GSH}$ - nmoles/g wet tissue; LPO - nmoles of malondialdehyde/mg protein.

\section{Animals}

Specific and pathogen free Wistar strain rats were chosen for this study. They were obtained and acclimatized to laboratory environment. They were housed in polypropylene cages with stainless steel grid covers and bedding material used is paddy or wheat husk. They were groomed in a controlled environment with feed and drinking water provided in polypropylene bottles. Experimental protocols were approved by Institutional Animal Ethics Committee (IAEC), which follows the guidelines of CPSCEA (Committee for the purpose of control and supervision of experiment on Animals) and the Guide for the care and use of laboratory animals. The IAEC approval number for this study was: Biochem: 005/2008 \& 01/003/10.

\section{Test Compound and dose administration levels}

Anti-carcinogenic test is performed in accordance to the guidelines of World Health Organization (WHO 2000) and the Organization of Economic Co-operation and Development (OECD) guideline for testing of chemicals (OECD, 2001). As the toxicity of the compound Garcinia mangostana is determined in earlier studies, the drug dose is chosen accordingly. Four groups of 6 rats each is used for the study. Group I: Normal rat received distilled water (1 ml/kg b.wt); Group II: Rats induced with diethylnitrosamine (DEN) (0.01\% DEN through drinking water upto 16 weeks); Group III: Rats induced with DEN (0.01\% DEN through drinking water upto 16 weeks and cotreated with Garcinia mangostana extract (400 mg/kg wt); Group IV: Rats treated with Garcinia mangostana extract (400 mg/kg wt) alone. Food and water were accessible ad libitum. Animals were sacrificed after 16 weeks and livers were dissected and then excised; samples of lobes were immersed in 4\% buffered formaldehyde for histological analysis. Counts of suspicious nodules on the liver surface were performed by macroscopic examination of the liver based on the following criteria: nodules with a diameter of $3 \mathrm{~mm}$ or more and a dysmorphic or dyschromic aspect; these counts were expressed as the number of nodules per gram of liver.

\section{Weight examination}

The weight of the animal is continuously monitored everyday for the first seven days and every alternate days for the rest 16 weeks. After euthanasia the weight of the liver, the main focus of the study was evaluated and studied for any abnormal gain or loss of weight from normal. This gives a preliminary confirmation regarding the adverse effects (if any) of the drug under test. ${ }^{25}$

\section{Biochemical Parameters}

Clinical biochemistry parameters especially the liver marker enzymes like AST (aspartate aminotransferase), ALT (alanine aminotransferase), Lactate Dehydrogenase (LDH) and ALP (alkaline phosphatase), GammaGlutamyl Transpeptidase, and 5' nucleotidases were estimated using commercially available diagnostic kits and semi-automatic analyzer.

\section{Antioxidants analysis}

Lipid peroxide content in liver tissues was determined by thiobarbituric acid reaction as described by Okhawa et $a l^{26}$ The levels of reduced glutathione (GSH) were measured according to the method of Moron et al, ${ }^{27}$ The activity of catalase was assayed by the method of Sinha. ${ }^{28}$ The activity of superoxide dismutase (SOD) was assayed by the method of Misra and Fridovich. ${ }^{29}$ 


\section{Statistical analysis}

Results will be expressed as mean \pm S.E.M. Statistical significance is determined by one-way analysis of variance (ANOVA) and post hoc least-significant difference test. The data obtained from acute toxicity studies will be analyzed using Student's paired t-test. P values less than 0.05 will be considered significant.

\section{RESULTS}

Continued oral administration of DEN did not induce any mortality to the animals, but showed drastic decrease in body weight. The extract group, DEN + extract and the control group however showed slight increase in body weight. This provides an initial evidence for the protective and therapeutic effect of the drug. Development of tumor in the liver causes significant difference in the weight of the organ. When the animals are excised and their liver weights are examined to the nearest accuracy possible, it exhibited very clear distinction and good therapeutic behavior of the drug (Table 1).

Cancer growth can be visible observed when the liver is excised with the number of nodules that are developed on the surface of the liver. The Control and GME group showed complete absence of hepatic nodules, the DEN fed group of animals showed the presence of large number of nodules while the DEN + GME group showed reduced number of nodules indicating the protective nature of the drug (Table 2).

The hepatic carcinoma induced group (group 2) produced severe liver injury as evidenced by the significantly increased levels of SGOT, SGPT, LDH GGT and 5' nucleotidase in serum, compared with that of the control as depicted in Figure 1. However, the DEN induced rats treated with GM extract showed an obvious decrease in liver marker enzyme levels showing its hepatoprotective effect.

The results of oxidative stress and anti-oxidant levels in experimentally induced and treated DEN were shown in Figure 2. Non enzymatic antioxidant such as GSH and anti-oxidant enzymes such as catalase and SOD were decreased significantly with simultaneous LPO increase in group 2 rats, compared to that of control group. The carcinoma treated with GM extract showed significant increase in the levels of antioxidants viz. GSH, catalase, SOD and decrease in LPO when compared with that of group 2 rats.

\section{DISCUSSION}

The initiation, development and progress of the cancer are one of the major domains to study and analyze the methodology in which tumor protection drug has to be produced. Also, when N- Diethyl nitrosamine (DEN) is administered to initiate cancer in Wistar rats, it was observed that the DEN produced cancerous effects on liver when administered continuously over a period of time of 45-60 days. DEN induced hepatic cells to turn carcinogenic and enhance the release of free radicals on a large scale that damage the surrounding cells. As the tumor developed, it was clearly observed that there was significant loss in their body weight and it was reflected in the abnormal increase in the tumorous nodules formed in the liver region which could be felt easily by human touch on the abdominal cavity. When all the animals are maintained under normal feed and environmental conditions, those which were fed with DEN alone showed drastic loss of weight, whereas those which were administered the DRUG along with DEN showed significant recovery from the damage due to free radicals. The extract Garcinia mangostana showed powerful anti-oxidant activity by which it reduced the effect of DEN on the liver cells and prevented the development of tumor cells and also the loss of weight of the animal. More continuous observation of the animal showed that the extract had clear therapeutic characteristics as the weight of the animal started to increase at the terminal stage of the study.

After excising the animal the liver of the animal was also weighed and it showed that the control and the extract group had no significant change in the weight of the liver and also DEN + Extract had shown only minor increase in the liver weight. DEN group of animals showed very high increase in the liver weight that clearly differentiates the damaging effect of the chemical and also the preventing effect of the drug. The drug's bio chemical effect was distinctly observed as the liver nodules were found in very least number in the group that was fed with both DEN and GME. DEN induces tumor nodules to develop as the carcinogenic cells extract the nutrition from surrounding cells, hence the number of hepatic nodules that were observed in the surface of the liver were extremely high when compared to that of the surface of the liver of the animal, which was fed with DRUG also.

The lipid peroxidation (LPO) level and level of enzymatic antioxidants in the liver tissue of control and experimental animals is presented in Figure 2. DEN administration produced a profound elevation (threefold) in the levels of MDA, as compared to control rats. Treatment with GML pericarp extract after DEN administration significantly decreased the levels of MDA, when compared with DEN induced rats. There was a significant decrease in the activity of both SOD and CAT upon administration of DEN in the liver tissue, which was restored back to normalcy upon treat- 
ment with GML pericarp extract. Administration of GML pericarp extract alone (Group IV) did not produce any significant alterations in most of the parameters investigated.

Lipid peroxidation, initiated in the presence of hydroxyl radicals resulting in the production of malondialdehyde (MDA), directly produces oxidative stress. ${ }^{30}$ Glutathione $(\mathrm{GSH})$ is a key player in reduction processes in the cell. ${ }^{31}$ It also plays a role in reduction of nucleotide tri phosphate (NTP) to deoxy nucleotide tri phosphate (dNTP) and in detoxification of endogenous and exogenous compounds, serves as a cofactor for various enzymes, stores and transports cysteine, and may be involved in cell cycle regulation and thermotolerance. ${ }^{32,33}$ Glutathione reductase (GR) is a gene encoding for an enzyme which reduces glutathione disulfide (GSSG) to the sulfhydryl form GSH, which is an important cellular antioxidant. ${ }^{34}$

Membrane lipids are easily susceptible to deleterious actions of reactive oxygen species. Measurement of lipid peroxidation is considered to be a convenient method to monitor oxidative membrane damage. ${ }^{35}$ There is a strong correlation between oxidative stress and the occurrence of liver cancer. Enhanced lipid peroxidation and/or a dysregulated antioxidant system have been associated with liver cancer in both experimental animal models and in humans. ${ }^{36}$ In the present study, a threefold increase in the levels of LPO observed in liver tissue of DEN administered rats is the consequence of oxidative stress caused by DEN resulting from peroxidative membrane damage. Free radicals mediated peroxidation of membrane lipids might have resulted in loss of membrane integrity and membrane damage, leading to its rupture and subsequent release of the cytosolic contents. Previous studies have shown Mangosteen to be an effective antioxidant under in vitro conditions. ${ }^{37}$ The fall in the levels of LPO observed after treatment with GML pericarp extract in the present study is suggestive of the fact that GML pericarp extract is successful in quenching the free radicals thereby inhibiting lipid peroxidation and protecting the membrane lipids from oxidative damage in the liver of rats. Hence GML pericarp extract acts as an effective antioxidant in in vivo conditions also.

SOD and CAT constitute a team of mutually supportive antioxidative enzymes, which provide protective defense against reactive oxygen species. ${ }^{38}$ During DEN induced hepatocellular carcinoma these enzymes are structurally and functionally impaired by free radicals resulting in liver damage. The restoration of the activities of antioxidant enzymes could be due to the ability of GML pericarp extract to scavenge reactive oxygen species within the lipid region of the membrane. Glutathione peroxidase (GPX) is a general name of enzyme family with peroxidase activity whose main biological role is to protect the organism from oxidative damage. The biochemical function of glutathione peroxidase is to reduce lipid hydroperoxides to their corresponding alcohols and to reduce free hydrogen peroxide to water. ${ }^{39}$ Consequently, the levels of GSH (a key player in reduction and detoxification processes), GR (reduces GSSG to GSH which is an important cellular antioxidant) and GPX (whose main biological role is to protect the organism from oxidative damage) ${ }^{39}$ decreased significantly in DEN induced HCC rats (Figure 2). The higher concentration of GSH in GML treated group than control could be attributed to the increase of GSSG-GSH transformation, by GR enzyme, to overcome the increased lipid peroxidation exerted by DEN.

DEN administration decreased the activities of these antioxidant enzymes in the liver tissue by about $50 \%$. The decline in the activities of these enzymes in the present study could be attributed to the excessive utilization of these enzymes in inactivating the free radicals generated during the metabolism of DEN. The restoration in the activities of these enzymes after GML pericarp extract administration indicates that GML acts as an effective antioxidant. GML possess phytochemicals like xanthones, phenols and other flavonoids, which have been shown to be effective in scavenging free radicals. ${ }^{39,40}$ Therefore by acting as an alternate radical scavenger, GML can replenish these antioxidant enzymes, thereby preventing oxidative stress. These results of our investigations are in accordance with that of Weecharangsan et al., ${ }^{41}$ who have reported the antioxidant activity of GML pericarp extract.

GM exhibits profound anti-oxidant abilities which reduces the levels of lipid peroxides and increases the levels of glutathione, SOD and CAT to near normal performance of the liver. The increase in the levels of these anti-oxidants is due to the drug that prevents the increase in levels of oxidation in the liver cells. The lipid peroxide levels are found to be high in the levels of the DEN alone group. The liver marker enzymes shows very high levels in the DEN alone group whereas the levels of the same are significantly reduced in the GME treated group which is again attributed to the reduction in the oxidation activity of the DEN. The foretold results experimentally prove that the Garcinia mangostana extract which had earlier been proven to be a powerful anti-oxidant is indeed a very powerful drug against the hepatic carcinoma.

\section{CONCLUSION}

GM exhibits profound anti-oxidant abilities which reduces the levels of lipid peroxides and increases the 
levels of glutathione, SOD and CAT to near normal performance of the liver. The increase in the levels of these anti-oxidants is due to the drug that prevents the increase in levels of oxidation in the liver cells. The lipid peroxide levels are found to be high in the levels of the DEN alone group. The liver marker enzymes shows very high levels in the DEN alone group whereas the levels of the same are significantly reduced in the GME treated group which is again attributed to the reduction in the oxidation activity of the DEN. The foretold results experimentally prove that the Garcinia mangostana extract which had earlier been proven to be a powerful anti-oxidant is indeed a very powerful drug against the hepatic carcinoma.

\section{CONFLICTS OF INTEREST}

Authors declared no conflict of interest.

\section{ACKNOWLEDGEMENTS}

The Authors are very much thankful to Dr. S. Porchelvan, M.Sc., M.B.A., PGDCA., PhD., Professor in Biostatistics, Saveetha Medical College \& Hospital, Saveetha University for assisting us in performing the statistical analyses.

\section{AUTHOR'S CONTRIBUTIONS}

VP proposed the idea of the study, participated in study design, performed biochemical analyses, participated in acquiring and managing the data and carried out data collection, interpretation of the data and contributed in preparation of manuscript, Contributed in preparation of draft manuscript. SKM participated in the design of the study, carried out various biochemical analyses, helped in preparation of draft manuscript. MJ participated in the design of the study and participated in performing the various biochemical analyses. CSG proposed the idea of the study and participated in study design. All authors read and approved the final manuscript.

\section{Highlights of Paper}

- Diethylnitrosamine (DEN), environmental carcinogen induces hepatocellular carcinoma in rats.

- DEN has been suggested to cause oxidative stress and cellular injury due to the enhanced formation of free radicals.

- The pericarp of the Garcinia mangostana Linn is a waste product; but the pericarp of Mangosteen fruit is rich in xanthones, anthocyanins and oligomeric proanthocyanidin.

- This study demonstrated the free radical scavenging potential, antioxidant, hepatoprotective and anti tumour activities of Garcinia mangostana Linn pericarp extract (GME).

- GME shows modulating effect by maintaining the antioxidants to normal status.

- Hence, this could be safely developed as a hepatoprotective adjuvant for hepatocellular carcinoma.

\section{Author Profile}

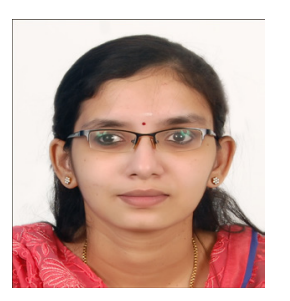

- Dr. Vishnu Priya V, holds PhD in Biochemistry and her thesis was highly commendable. She published around 25 research papers in various national and international, indexed journals to her credit. She was the recipient of IFCC Fellowship in Brazil, DNA Topology fellowship in Japan. She has won "Young achievers award", best research paper award in national conferences and best teacher award. She is guiding many students for their research projects. She completed TPRM from university of McMaster. She attended many national and international conferences and chaired various session in international arena.

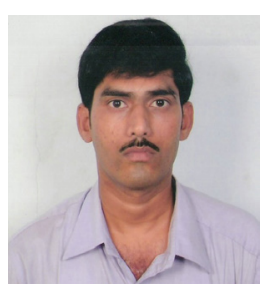

- Dr. Surapaneni Krishna Mohan holds PhD (Medical) in Biochemistry and presently working as Associate Professor of Biochemistry \& Vice Principal in Saveetha Medical College \& Hospital, Saveetha University, Chennai, India. His research interests include biochemical studies in Non Alcoholic Steatohepatitis (NASH) and other liver diseases, Operations research in population health, public health informatics and research in health professions education. He was awarded the prestigious "Chartered Scientist (CSci)" qualification, which represents a single chartered mark for all scientists, recognizing high levels of professionalism and competence in science, by "The Science Council", UK and "The Royal Society of Chemistry (RSC), UK. Also, he is a "Certified Clinical Chemist" by National Registry of Certified Chemists (NRCC), USA. He published several scientific research papers in reputed national and inter- 
national indexed journals to his credit. He is the life member of many professional national and international scientific societies / bodies. He has received several international travel grants and fellowships to present his work in several international conferences and workshops. He has received many awards like "Young Investigator Award" \& "Young Scientist" award, to name a few, for his research work on international platform.

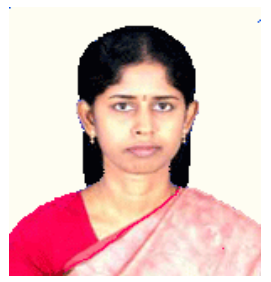

- Dr. Mallika Jainu, is an Assistant professor at the Dept of Biomedical Engineering, SSN College of Engineering, Chennai. Her research interest in the area of Pharmacology, Toxicology \& Bioengineering. She has 43 nos of Journal publication, 35 nos of abstract, 2 nos of book chapter. Acting as an investigator in two ongoing funded projects.

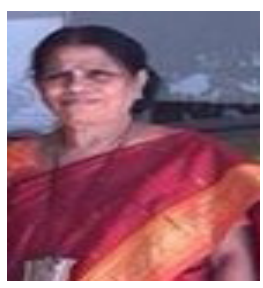

- Dr. V S Schandra Sada Gopan holds PhD in Biochemistry and retired from Govt. Stanley Medical College, Chennai. After her retirement she worked as Professor of Biochemistrry in medical colleges in India and presently she is working as Professor \& Head, Department of Biochemistry, Priyadarshini Dental College \& Hospitals, Pandur, Thiruvallur. She holds more than three decades of teaching experience in medical colleges teaching medical students. She is the recognized as $\mathrm{PhD}$ guide by various universities to guide the students to pursue their $\mathrm{PhD}$. She published many research papers in various national and international, indexed journals to her credit. She attended many national and international conferences and chaired various session in international arena. Also, she received many awards for her excellence in teaching and research at various national and international platforms.

\section{REFERENCES}

1. Wogan GN. Dietary risk factors for primary hepatocellular carcinoma. Cancer Detect Prev. 1989; 14(2): 209-11.

2. Guyton KZ, Kensler TW. Oxidative mechanisms in carcinogenesis. Br Med Bull. 1993; 69(3): 523-544.

3. Farber E. The multistep nature of cancer development. Cancer Res. 1984; 44(10): 4217-23.

4. Akintonwa DA. The derivation of nitrosamines from some therapeutic amines in the human environment. Ecotoxicol Environ Saf. 1985; 9(1): 64-70.

5. Coker HA, Thomas AE, Akintonwa A. Determination of the total level of nitrosamines in select consumer products in Lagos area of Nigeria. Bull Environ Contam Toxicol. 1991; 47(5): 706-10.

6. Tricker AR, Pfundstein B, Theobald E, Preussman R, Spiegehalder B. Mean daily intake of volatile $N$-nitrosamines from foods and beverages in West Germany in 1989-90. Food Chem Toxicol. 1991; 29(11): 729-32.

7. Fine D H, Ross R, Rounbehler DP, Silvergleid A, Song L. Formation in vivo of volatile $\mathrm{N}$-nitrosamines in man after ingestion of cooked bacon and spinach. Nature 1977; 265(1): 753-5.

8. Zhou Li X, Guan XP, Wang YX. Magnetic resonance imaging of hepatocellular carcinoma induced by diethylnitrosamine in Sprague-Dawley rats. Hepatobiliary Pancreat Dis Inf. 2005; 4(3): 427-32.

9. Kim MR, Kim HS, Lee MS, Lee MJ, Jang JJ. Cell cycle protein profile of the hepatic stellate cells (HSCs) in dimethylnitrosamine-induced rat hepatic fibrosis. Exp Mol Med. 2005; 37(4): 335-342.

10. Verna L, Whysner J, Williams GM. N-nitrosodiethylamine mechanistic data and risk asseessment: bioactivation, DNA-adduct formation, mutagenicity, and tumor initiation. Pharmacol Ther. 1996; 71(1): 57-81.

11. Cerutti PA. Response modification creates promotability in multistage carcinogenesis. Carcinogenesis 1988; 9(4): 519-26.

12. Krishna Mohan Surapaneni, Chandra Sada Gopan VS. Status of Lipid peroxidation and anti oxidant enzymes in patients with carcinoma of breast. Journal of Medical Sciences Research 2007; 15(1): 21 -24.

13. Trush MA, Kensler TW. Role of free radicals in carcinogenic activation, in: H. Sies (Ed.), Oxidative Stress: Oxidants and Antioxidants, Academic Press, London; 1991, pp. 277-318.

14. Witz G. Active oxygen species as factors in multistage carcinogenesis. Proc Soc Exp Biol Med. 1991; 198(2): 675-82.
15. Frenkel K. Carcinogen mediated oxidant formation and oxidative DNA damage. Pharmacol Ther. 1992; 13(1): 127-66.

16. Sharmila Upadhya, Subramanya Upadhya, Krishna Mohan, Vanajakshamma, Mamatha Kunder, Seema Mathias. Oxidant - Anti-oxidant status in colorectal cancer patients - before and after treatment. Indian J Clin Biochem. 2004; 19(2): 80-3.

17. Salim AS. Removing oxygen-derived free radicals delays hepatic metastases and prolongs survival in colonic cancer: a study in rat. Oncology 1991; 49(1): 58-62.

18. Perchellet JP, Perchellet EM. Anti-oxidants and multistage carcinogenesis in mouse skin. Free Radic Biol Med. 1989; 7(4): 377-408.

19. Aruoma IO. Free radicals and antioxidants strategies in sports. J Nutr Biochem 1994; 5(8): 370-81.

20. Williams GM, Furuya K. Distinction between liver neoplasm promoting and syncarcinogenic effects demonstrated by exposure to phenobarbital or diethylnitrosamine either before or after $\mathrm{N}$-2-fluorenylacetamide. Carcinogenesis $1984 ; 5(2): 171-4$.

21. Hartwell JL. Types of anticancer agents isolated from plants. Cancer Treat Rep. 1976; 60(8): 1031-67.

22. Primchanien Moongkarndi, Nuttavut Kosema, Sineenart Kaslungka, Omboon Luanratana, Narongchai Pongpan, Neelobol Neungton. Antiproliferation, anti oxidation and induction of apoptosis by Garcinia mangostana (mangosteen) on SKBR3 human breast cancer cell line. Journal of Ethnopharmacology 2004; 90(1): 161-6.

23. José Pedraza-Chaverri, Noemí Cárdenas-Rodríguez, Marisol Orozco-lbarra, Jazmin M, Pérez-Rojas. Medicinal properties of mangosteen (Garcinia mangostana). Journal of food and toxicology 2008; 46(10): 24-7.

24. Limei Yu, Mouming Zhao, Bao Yang, Qiangzhong Zhao, Yueming Jiang. Phenolics from hull of Garcinia mangostana fruit and their antioxidant activities, Elsevier; November 2006.

25. Helle Andersen, Søren Larsen, Henrik Spliid, Niels Dyhr Christensen. Multivariate statistical analysis of organ weights in toxicity Studies. Toxicology 1999; 136(2): 67-77.

26. Okhawa $\mathrm{H}$, Ohishi $\mathrm{N}$, Yagi K. Assay for lipid peroxide in animal tissues by thiobarbituric acid reaction. Anal Biochem. 1979; 95(2): 351-7. 
27. Moron NS, Depierre JW, Mannervik B. Levels of glutathione, glutathione reductase and glutathione-Stransferase activities in rat lung and liver. Biochem Biophys Acta. 1979; 582(1): 67-72.

28. Sinha AK. Colorimetric assay of catalase. Anal Biochem. 1972; 47(2): 389-94.

29. Misra HP, Fridovich I. The role of superoxide anion in the autooxidation of epinephrine and a simple assay for superoxide dismutase. J Biol Chem. 1972; 247(10): 3170-5

30. Surapaneni KM, Vishnu Priya V. Status of lipid peroxidation, glutathione, ascorbic acid, vitamin $\mathrm{E}$ and anti-oxidant enzymes status in neonatal jaundice patients. Journal of Clinical and Diagnostic Research 2008; 2(3): 827-32.

31. Surapaneni Krishna Mohan, Vishnu Priya V. Serum paraoxonase activity, protein oxidation and lipid peroxidation levels in patients with coronary artery disease. Asian J Exp Biol Sci. 2010; 1(2): 254 - 61.

32. Champe PC, Harvey RA, Ferrier D. Biochemistry. Nitrogen Metabolism (Fourth ed. by Scogna, K.) 2008; 27(23): 245-306.

33. Leelavinothan Pari D, Rosalin Amali. Protective role of tetrahydrocurcumin (THC) an active principle of turmeric on chloroquine induced hepatotoxicity in rats. J Pharm Pharmaceut Sci. 2005; 8(1): 115-23.

34. Surapaneni KM, Vishnu Priya V. Anti-oxidant Enzymes and Vitamins in Gestational Diabetes. Journal of Clinical and Diagnostic Research 2008 Oct; 2(5): 1081-5.
35. Surapaneni Krishna Mohan, Vishnu Priya V. Serum Total Sialic Acid, Lipid peroxidation and glutathione Reducatse levels in patients with Rheumatoid Arthritis. Turk J of Med Sci. 2010; 40(4): 537-40.

36. Zhao L, He J, Wang X, Zhang L. Nitric oxide protects against polyethylene glycol-induced oxidative damage in two ecotypes of reed suspension cultures. J Plant Physiol. 2008; 165(2): 182-91.

37. Ngawhirunpat T, Opanasopi P, Sukma M, Sittisombut C, Kat A, Adachi I. Capturing circulating tumor cells of hepatocellular carcinoma. Pharm Biol. 2010; 48(1): 55-62.

38. Prasanna Galhena A, Ira Thabrewa Mayuri G, Tammitiyagodageb, Vasanthi A. Anti-hepatocarcinogenic Ayurvedic herbal remedy reduces the extent of diethylnitrosamine-induced oxidative stress in rats. Pharmacognosy Magazine 2009; 5(17): 19-27.

39. Jung HA, Su BN, Keller WJ, Mehta RG, Kinghorn AD. Anti-oxidant xanthones from the pericarp of Garcinia mangostana (Mangosteen). J Agric Food Chem. 2006; 54(6): 2077-82.

40. Fabiola Gutierrez-Orozco, Mark Failla L. Biological Activities and Bioavailability of Mangosteen xanthones: A Critical Review of the Current Evidence. Nutrients 2013; 5(8): 3163-83. doi:10.3390/nu5083163

41. Weecharangsan $W$, Opanasopit $P$, Sukma M, Ngawhirunpat $T$, Sotanaphun $U$, Siripong P. Anti-oxidative and Neuroprotective Activities of Extracts from the Fruit Hull of Mangosteen (Garcinia mangostana Linn.). Med Princ Pract. 2006; 15(4): 281-7. 